\title{
Synergistic induction of growth arrest and apoptosis of human myeloma cells by the IL-6 super-antagonist Sant7 and Dexamethasone
}

\section{Dear Editor,}

The results of the medical treatment of multiple myeloma (MM) have not substantially improved during the last 30 years. ${ }^{1}$ The combination of an alkylating agent and a glucocorticoid remains the most used therapeutic regime. Recently, high-dose chemotherapy followed by autologous stem cell transplantation has achieved high response rates. ${ }^{2}$ However, none of the strategies thus far available appears effective in avoiding the persistence of residual disease, the development of drug-resistant myeloma cells and the consequent fatal relapse.

Interleukin-6 (IL-6) of paracrine ${ }^{3,4}$ but also of autocrine ${ }^{5}$ origin is known to sustain the survival and proliferation of MM cells, ${ }^{6}$ and to protect them from apoptosis induced by a variety of agents, including glucocorticoids. ${ }^{7,8} \mathrm{IL}-6$ receptor antagonists, ${ }^{9,10}$ which prevent the assembly of functional IL-6 receptor complexes, may be instrumental in the development of more effective therapeutic approaches for MM.

This study was aimed to evaluate whether inhibition of the IL- 6 signalling pathway by the IL- 6 super-antagonist Sant $7^{11}$ may enhance the cytotoxic effect of Dexamethasone (Dex) on myeloma cells in vitro. We sought to generate a scenario resembling an in vivo situation where the growth of MM cells is under the influence of both paracrine and autocrine IL-6. To this end: (i) we established an autocrine subclone of the IL-6-dependent human myeloma cell line $X G-1^{12}$ by serial passages in low concentrations $(0.1 \mathrm{ng} / \mathrm{ml})$ of $\mathrm{IL}-6$. These cells, named XG-1a, secrete detectable amounts of IL-6 and, when cultured in the absence of exogenous factor, they continue to proliferate after an initial lag (not shown); (ii) we cultured the XG-1a cells in the presence of exogenous IL-6 $(0.2 \mathrm{ng} /$ $\mathrm{ml})$, and exposed them to Sant7 $(200 \mathrm{ng} / \mathrm{ml})$, Dex $\left(10^{-5} \mathrm{M}\right)$, or the combination of the two agents. The number of viable cells, the percentage of cells in S-phase and the rate of apoptosis was measured at different time intervals. As shown in Figure 1A, addition of Dex caused a decrease in the number of viable XG-1a cells, but did not modify the entry in the S-phase and induced only a mild increase in the percentage of hypodiploid cells. Addition of Sant7 resulted in a decrease in the number of viable cells, a significant reduction of S-phase, and a moderate increase in the fraction of hypodiploid cells, similar to that elicited by Dex. The association of Sant7 and Dex displayed a strong synergistic action in inhibiting cell proliferation and entry in $\mathrm{S}$-phase, as well as in inducing apoptosis.

To verify that the hypodiploid DNA content detected by Propidium-iodide $(\mathrm{PI})$ staining did reflect apoptotic death, XG-1a cells treated with Sant7 and Dex were also analyzed by the Mebstain, a flow cytometric assay based on a modification of the TUNEL technique. As shown in Figure

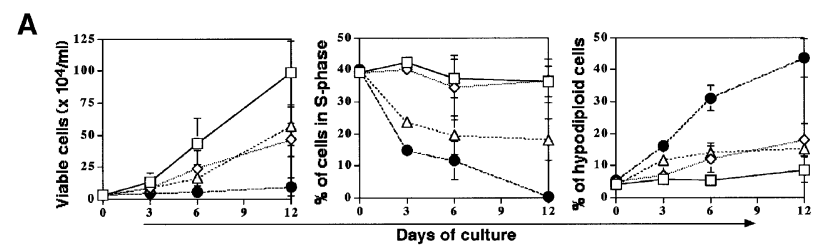

B

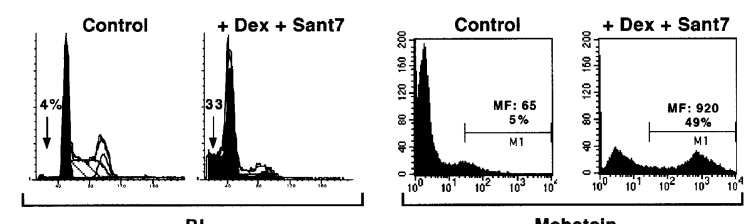

C

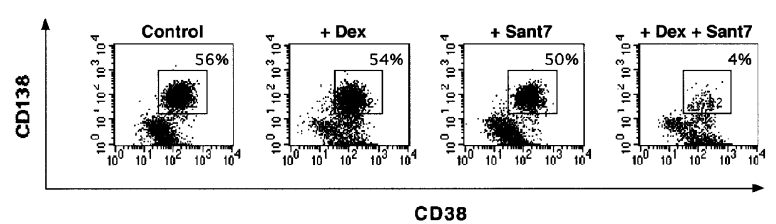

Figure 1 Effect of Sant7 and/or Dex on proliferation and apoptosis of XG-1a cells. (A) Viable cells as determined by trypan blue exclusion were counted in a Bürker chamber. For analysis of cell-cycle and determination of DNA content, a PI-staining kit (CycleTEST ${ }^{\mathrm{TM}}$ PLUS, Becton Dickinson) was used following the instructions furnished by the manufacturer. Flow cytometry was carried out in a FACScan flow cytometer (Becton Dickinson) and data were analyzed using a software for DNA analysis (Verity Software House). Apoptotic cells were detected as hypodiploid populations. ${ }^{13}$ Data are reported as a mean \pm S.D. of at least three separate experiments. The effect induced by Dex+Sant7 was statistically significative as compared to either compound alone $(P<0.05$; $t$-test). Symbols used are: $(\square)$, untreated cells; $(\diamond)$, Dex; $(\triangle)$, Sant7; $(\mathbf{O})$, Dex+Sant7. (B) Flow cytometric profiles of a representative experiment in which apoptotic cell death was analyzed in parallel by PI-staining and the Mebstain method. The latter, which measures the incorporation of fluorescein-dUTP (FITC-dUTP), was performed using a commercial kit (Immunotech) according to the instructions of the manufacturer. Analysis was carried out by flow cytometry using the CellQuest program (Becton Dickinson). The percentage of apoptotic cells (\%), and the mean fluorescence intensity (MF), are indicated. (C) Effect of Sant7 (200 ng/ $\mathrm{ml}$ ) and/or Dex $\left(10^{-5} \mathrm{M}\right)$ on co-cultures of purified $\mathrm{CD} 34^{+}$haematopoietic progenitors and XG-1a myeloma cells. Mobilized peripheral blood CD $34^{+}$cells were isolated from leukaphereses of tumour patients treated with high-dose chemotherapy and granulocyte colony stimulating factor, using the CD34 Progenitor Cell Isolation Kit (Miltenyi Biotech). The purity of the cells thus isolated was over $90 \% .2 \times 10^{5} \mathrm{CD}_{3} 4^{+}$and $2 \times 10^{4} \mathrm{XG}-1$ a cells $/ \mathrm{ml}$ were grown in the presence of interleukin-3, Flt3 ligand and stem cell factor to promote the survival of $\mathrm{CD}_{3} 4^{+}$cells and of IL- 6 to support the growth of XG-1a. Flowcytometry profiles of CD38 and CD138 (syndecan-1) expression were performed after 12 days of culture. Haematopoietic progenitors and myeloma cells were scored as CD $38^{+} / \mathrm{CD} 138^{-/ \mathrm{dim}}$ and $\mathrm{CD} 38^{+} / \mathrm{CD} 138^{\text {bright }}$ respectively. Data shown are from a representative experiment 
$1 \mathrm{~B}$, the Mebstain analysis yelded results similar to those obtained with the PI staining.

The data shown in Figure 1 suggest that the synergistic action of Sant7 and Dex overcomes the IL-6-mediated cell resistance to glucocorticoids, thereby promoting growth arrest and apoptosis of myeloma cells. Thus, Sant7 might be advantageously associated to Dex in the treatment of $\mathrm{MM}$ in vivo and/or in ex-vivo purging of mobilised stem cells to be transplanted following high-dose chemotherapy. However, the question remains whether administration of Sant7 may result in adverse effects on normal haematopoiesis.

A first series of experiments (data not shown) demonstrated that treatment of human mobilised $\mathrm{CD} 34^{+}$cells with Sant7 did not show any detectable effect on cell viability, proliferation, antigenic profile and development of haematopoietic colonies in response to cytokines, whereas Dex induced a $20-30 \%$ decrease in the number of colonies and of cells in S-phase, not accompanied by detectable apoptosis. The combination of Dex and Sant7 produced effects indistinguishable from those of Dex alone, confirming the lack of interference of Sant7 with the hemopoietininduced survival, growth and differentiation of $\mathrm{CD} 34^{+}$cells and lending support to the idea that Sant7 can be safely associated to Dex in the treatment of MM. To further confirm the selective cytotoxic effect of the association of Sant7 and Dex on MM cells, co-culture experiments were performed to simulate an ex-vivo condition in which myeloma cells contaminate a population of normal CD34 ${ }^{+}$ progenitors in the presence of cytokines that promote the survival of myelopoietic progenitors and of IL-6. As shown in Figure 1C, incubation with Sant7 and Dex for 12 days drastically abated the relative number of myeloma cells, whereas either agent appeared to be completely ineffective when used alone.

Taken together, our results indicate that the IL-6 superantagonist Sant7 strongly enhances the cytotoxic effects of Dex on MM cells without interfering with the normal human haematopoiesis. The combination of an IL- 6 antagonist and a glucocorticoid, which shows a potent inhibitory effect on cell growth, could represent a successful approach to overcome the myeloma cell resistance and may be evaluated for the eradication of minimal residual disease after cytotoxic chemotherapy. In addition, the autocrine XG1a subclone could provide a valuable model to dissect the molecular mechanisms underlying the synergism between Sant7 and Dex in inducing apoptosis of MM cells and to identify cross-talks between the pathways which mediate the effects of the two molecules.

Work supported in part by funds from: Associazione Italiana per la Ricerca sul Cancro; Regione Calabria (POP 94/99); MURST-CNR Biotechnology Program L. 95/95; MURST (Cofin 98) and CNR (Special Project Biotechnology).

\section{$P$ Tassone ${ }^{1}$, S Forciniti ${ }^{1}$, E Galea $^{1}, R$ Savino $^{2}$, MC Turco ${ }^{1}, P$ lacopino ${ }^{3}, P$ Tagliaferri ${ }^{1}$, G Morrone $^{1,4}$ $G$ Ciliberto ${ }^{2}$ and $S$ Venuta $^{\star, 1}$}

${ }^{1}$ Dipartimento di Medicina Sperimentale e Clinica, Università di Catanzaro 'Magna Graecia', Catanzaro, Italy; 'Istituto di Ricerche di Biologia Molecolare, P. Angeletti, Via Pontina, km 30,600, Pomezia, Rome, Italy; ${ }^{3}$ Dipartimento di Ematologia-Oncologia, Azienda Ospedaliera 'Bianchi-Melacrino-Morelli', Reggio Calabria, Italy; ${ }^{4}$ CEINGE Advanced Biotechnology, Napoli, Italy.

${ }^{*}$ Corresponding author: Prof. Salvatore Venuta, Dipartimento di Medicina Sperimentale e Clinica, Università di Catanzaro 'Magna Graecia', Catanzaro, Italy. Tel: +39.0961.775007; Fax: +39.0961.775373; E-mail: oncologia@unicz.it

1. Alexanian R and Dimopoulos M (1994) New Engl. J. Med. 330: 484-489

2. Attal M, et al. (1996) New Engl. J. Med. 335: 91-97

3. Klein B, et al. (1989) Blood 73: 517-526

4. Caligaris-Cappio F, et al. (1991) Blood 77: 2688-2693.

5. Kawano M, et al. (1988) Nature 332: 83-85

6. Klein B, et al. (1995) Blood 85: 863-872

7. Hardin J, et al. (1994) Blood 84: 3063-3070

8. Grigorieva I, et al. (1998) Exp. Hematol. 26: 597-603

9. Savino R, et al. (1994) EMBO J. 13: 1357-1367

10. Sporeno E, et al. (1996) Blood 87: 4510-4519

11. Demartis A, et al. (1996) Cancer Res. 56: 4213-4218

12. Zhang X, et al. (1994) Blood 83: 3654-3663.

13. Darzynkiewicz Z, et al. (1992) Cytometry 13: 795-808 